\title{
Adams Operations for Projective Modules over Group Rings
}

\author{
BERNHARD KÖCK \\ Math. Institut II der Universität Karlsruhe, D-76128 Karlsruhe \\ e-mail: bk@ma2s2.mathematik.uni-karlsruhe.de
}

\begin{abstract}
Let $R$ be a commutative ring, $\Gamma$ a group acting on $R$, and let $k \in \mathbb{N}$ be invertible in $R$. Generalizing a definition of Kervaire we construct an Adams operation $\psi^{k}$ on the Grothendieck group and on the higher $K$ theory of projective modules over the twisted group ring $R \# \Gamma$. For this we use generalizations of Atiyah's cyclic power operations and shuffle products in higher $K$-theory. For the Grothendieck group we show that $\psi^{k}$ is multiplicative and that it commutes with base change, with the Cartan homomorphism, and with $\psi^{l}$ for any other $l$ which is invertible in $R$.
\end{abstract}

\section{Introduction}

On the classical ring of characters $K_{0}(\overline{\mathbb{Q}} \Gamma)$ of a finite group $\Gamma$ the $k$-th Adams operation $\psi^{k}$ is defined by

$$
\psi^{k}(\chi)(\gamma):=\chi\left(\gamma^{k}\right)
$$

(for any character $\chi \in K_{0}(\overline{\mathbb{Q}} \Gamma)$ and any $\gamma \in \Gamma$ ) or, equivalently, as the $k$-th Newton polynomial in the exterior power operations $\lambda_{1}, \ldots, \lambda_{k}$.

For the Grothendieck group $K_{0}(\mathbb{Z} \Gamma)$ of projective modules over the integral group ring $\mathbb{Z} \Gamma$ Fröhlich has given the following description, the so-called Hom-description:

$$
\frac{K_{0}(\mathbb{Z} \Gamma)}{\langle\text { free ones }\rangle} \cong \frac{\operatorname{Hom}_{G}\left(K_{0}(\overline{\mathbb{Q}} \Gamma), \mathcal{I}(\overline{\mathbb{Q}})\right)}{\operatorname{Hom}_{G}\left(K_{0}(\overline{\mathbb{Q}} \Gamma), \overline{\mathbb{Q}}^{\times}\right) \cdot \operatorname{Det}(\mathcal{U}(\mathbb{Z} \Gamma))}
$$

Here $G$ denotes the absolute Galois group $\operatorname{Gal}(\overline{\mathbb{Q}} / \mathbb{Q}), \mathcal{I}(\overline{\mathbb{Q}})$ the idele group of $\overline{\mathbb{Q}}$, Det the generalized determinant map, and $\mathcal{U}(\mathbb{Z} \Gamma)$ the group of unit ideles of $\mathbb{Z} \Gamma$ (see $[\mathrm{F}]$ for precise definitions and proofs). In [CNT] Cassou-Noguès and Taylor have shown that (for odd $k$ ) the homomorphism induced by $\psi^{k}$ on the numerator leaves the denominator of this formula invariant, i. e. $\psi^{k}$ induces a homomorphism $\psi_{k}$ on $K_{0}(\mathbb{Z} \Gamma) /\langle$ free ones $\rangle$. This paper grew out of the desire to describe this homomorphism $\psi_{k}$ in algebraic terms, i. e. to express $\psi_{k}$ in terms of power operations. Investigating the proofs of Fröhlich and Cassou-Noguès/Taylor one is led to the conjecture that there exist Adams operations on the Whitehead group $K_{1}\left(\mathbb{Z}_{p} \Gamma\right)$ of the group ring of $\Gamma$ over the $p$-adic integers $\mathbb{Z}_{p}$. The aim of this paper is to prove this conjecture in the following much more general setting.

Let $R$ be a commutative $\Gamma$-ring and let $k \in \mathbb{N}$ be invertible in $R$. Then for each $q \geq 0$ there exists an algebraically defined, additive Adams operation $\psi^{k}$ on Quillen's $q$-th $K$-group $K_{q}(R \# \Gamma)$ associated with the exact category of projective modules over the twisted group ring $R \# \Gamma$. 
To construct $\psi^{k}$ we generalize Kervaire's construction of $\psi^{k}$ on $K_{0}(K \Gamma)$ for a (finite) field $K$ (cf. [Ke]). In particular our definition is based on generalizations of Atiyah's cyclic power operations (cf. formula (2.7) of [At]). It uses shuffle products in higher $K$-theory (cf. [Ko2]) and Grayson's construction of power operations on higher $K$-theory (cf. [Gr1]).

We show that the $k$-th Adams operation $\psi^{k}$ on the Grothendieck group $K_{0}(R \# \Gamma)$ is multiplicative and that it commutes with the Cartan homomorphism $c: K_{0}(R \# \Gamma) \rightarrow K_{0}(\Gamma, R)$, with base change, and with the Adams operation $\psi^{l}$ for any other $l$ which is invertible in $R$. Furthermore we formulate a conjectural relationship between cyclic powers and exterior powers which one should be able to prove in representation theory and which would suffice to prove that $\psi^{k}$ commutes with the Cartan homomorphism $c: K_{q}(R \# \Gamma) \rightarrow K_{q}(\Gamma, R)$ in higher $K$-theory. Here $K_{q}(\Gamma, R)$ denotes Quillen's $q$-th $K$-group associated with $R$-projective $R \# \Gamma$-modules. Finally, if the characteristic of $R$ is a prime number, we define an Adams operation $\psi^{k}$ on $K_{q}(R \# \Gamma)$ for an arbitrary $k \in \mathbb{N}$ by combining the above construction with the base change homomorphism induced by the Frobenius endomorphism of $R$.

For $k$ coprime to the order $|\Gamma|$ of $\Gamma$ let $k^{\prime}$ be the inverse of $k$ modulo $|\Gamma|$. Then $\psi^{k^{\prime}}$ on $K_{0}(\overline{\mathbb{Q}} \Gamma)$ is the adjoint of $\psi^{k}$ with respect to the classical character pairing

$$
K_{0}(\overline{\mathbb{Q}} \Gamma) \times K_{0}(\overline{\mathbb{Q}} \Gamma) \rightarrow \mathbb{Z}
$$

(cf. the proof of Théorème 7-5 of $[\mathrm{CNT}]$ ). I believe that the Adams operations defined here are adjoints (in a sense which has still to be made precise) of those defined by Cassou-Noguès and Taylor. Having proved such an interpretation the Adams-Riemann-Roch theorem of [Ko1] and [Ko3] (for $K_{1}$ ) would presumably lead to an algebro-geometric explanation of the formula of Burns and Chinburg in $[\mathrm{BC}]$ where they compute the Adams operation of Cassou-Noguès and Taylor applied to certain ambiguous ideals in a tame Galois extension $K / \mathbb{Q}$ with $\operatorname{Gal}(K / \mathbb{Q})=\Gamma$. These topics will be the objects of further investigations.

I would like to thank D. Burns, T. Chinburg and M. Taylor for their encouraging interest in this project, and especially D. Burns for bringing the paper [BC] to my attention. Furthermore I would like to thank my colleagues at Karlsruhe for discussing several questions during the preparation of this paper.

\section{The Descent Construction}

Let $l$ be a prime number, $C$ a (multiplicative) cyclic group of order $l$ and $c \in C$ a fixed generator. The group $G:=(\mathbb{Z} / l \mathbb{Z})^{\times}$acts on $C$ by group automorphisms in the usual way and we denote by $C \rtimes G$ the corresponding semidirect product (defined by $\left(c^{a}, \sigma\right) \cdot\left(c^{b}, \tau\right):=$ $\left(c^{a+\sigma b}, \sigma \tau\right)$ for all $\left.a, b \in \mathbb{Z} / l \mathbb{Z}, \sigma, \tau \in G\right)$.

If in a commutative ring $R$ the prime number $l$ is invertible and if $R$ contains a primitive $l$-th root of unity $\zeta$, then, as is well-known, any module $V$ over the group ring $R C$ decomposes into the direct sum $V=\oplus_{a \in \mathbb{Z} / l \mathbb{Z}} V_{a}$ of "eigenspaces" $V_{a}:=\left\{v \in V: c(v)=\zeta^{a} v\right\}$. The object of this section is to construct an analogous decomposition for $V$ in the case when the assumption " $R$ contains a primitive $l$-th root of unity" is replaced by the following assumption: There is an action of $G$ on $V$ which together with the given action of $C$ on $V$ induces an action of $C \times G$ on $V$. This construction generalizes the descent argument of Kervaire given in $\S 5$ of [Ke] and will be needed in the following sections in the even more general situation when $V$ in addition carries a semilinear action of a further group $\Gamma$. 
So let $\Gamma$ be an (arbitrary, abstract) group, $R$ a commutative ring such that $l$ is invertible in $R$, $\Gamma \times R \rightarrow R$ an action of $\Gamma$ on $R$ by ring automorphisms and let $R \# \Gamma$ be the corresponding twisted group ring. Let $\Phi_{l}:=1+\ldots+X^{l-1} \in R[X]$ be the $l$-th cyclotomic polynomial and $S:=R[X] /\left(\Phi_{l}\right)$. Then the residue class $\zeta$ of $X$ is a primitive $l$-th root of unity in $S$ and the group $G$ acts on $S$ by $R$-algebra automorphisms given by $\sigma(\zeta):=\zeta^{\sigma}$ for $\sigma \in G$.

Now let $V$ be a $(C \rtimes G)$-module over $R \# \Gamma$. The generator $c$ of $C$ induces an $S$-linear and $R \# \Gamma$-linear automorphism of $S \otimes_{R} V$ which will be denoted by $c$ again. For any $a \in \mathbb{Z} / l \mathbb{Z}$ the eigenspace $\left(S \otimes_{R} V\right)_{a}:=\left\{x \in S \otimes_{R} V: c(x)=\zeta^{a} x\right\}$ is an $S$ - and $R \# \Gamma$-submodule of $S \otimes_{R} V$. Now we define an $S$-semilinear and $R \# \Gamma$-linear action of $G$ on $S \otimes_{R} V$ by $\sigma(\alpha \otimes v):=\sigma(\alpha) \otimes \sigma(v)$ for $\sigma \in G, \alpha \in S$ and $v \in V$.

Lemma 1. For each $a \in \mathbb{Z} / l \mathbb{Z}$ the eigenspace $\left(S \otimes_{R} V\right)_{a}$ is stable under this $G$-action.

Proof. For each $\sigma \in G$ we have $c \circ \sigma=\sigma \circ c^{\left(\sigma^{-1}\right)}$ in the semidirect product $C \rtimes G$. Hence for any $x \in\left(S \otimes_{R} V\right)_{a}$ we have $c(\sigma(x))=\sigma\left(c^{\left(\sigma^{-1}\right)}(x)\right)=\sigma\left(\zeta^{a \sigma^{-1}} x\right)=\zeta^{a} \sigma(x)$ in $S \otimes_{R} V$ and $\sigma(x)$ is contained in $\left(S \otimes_{R} V\right)_{a}$ again.

Definition. For any $a \in \mathbb{Z} / l \mathbb{Z}$ let

$$
F_{a}(V):=\left(S \otimes_{R} V\right)_{a}^{G}
$$

be the $R \# \Gamma$-submodule of $\left(S \otimes_{R} V\right)_{a}$ consisting of the $G$-invariant elements in $\left(S \otimes_{R} V\right)_{a}$.

Obviously, the association $V \mapsto F_{a}(V)$ defines a functor

$$
F_{a}:((C \rtimes G) \text {-modules over } R \# \Gamma) \rightarrow(R \# \Gamma \text {-modules }) .
$$

Proposition 1. The direct sum $\oplus_{a} F_{a}$ of the functors $F_{a}, a \in \mathbb{Z} / l \mathbb{Z}$, is isomorphic to the forgetful functor.

Proof. Let $V$ be a $(C \rtimes G)$-module over $R \# \Gamma$. On the $R \# \Gamma$-module $\operatorname{Maps}(G, V)$ the group $G$ acts via left translation on $G$ and via the given action on $V$. We will construct natural $R \# \Gamma$-isomorphisms

$$
\oplus_{a} F_{a}(V) \cong\left(S \otimes_{R} V\right)^{G} \cong \operatorname{Maps}_{G}(G, V) \cong V
$$

and these prove the proposition. The first isomorphism comes from Lemma 1 and the fact that the elements $e_{a}:=\frac{1}{l} \sum_{i=0}^{l-1} \zeta^{-a i} c^{i}, a \in \mathbb{Z} / l \mathbb{Z}$, form a complete system of orthogonal idempotents in the group ring $S C$ with $e_{a}\left(S \otimes_{R} V\right)=\left(S \otimes_{R} V\right)_{a}$ for all $a \in \mathbb{Z} / l \mathbb{Z}$. The second isomorphism is the restriction of the $G$-equivariant map $\operatorname{Maps}(G, V) \rightarrow S \otimes_{R} V, f \mapsto \sum_{\sigma \in G} \sigma(\zeta) \otimes f(\sigma)$ which is bijective because $\zeta$ is a normal basis element for the Galois extension $S / R$. The third isomorphism is the map $V \rightarrow \operatorname{Maps}_{G}(G, V), v \mapsto(\sigma \mapsto \sigma(v))$, which obviously is bijective.

\section{Corollary.}

a) The functors $F_{a}, a \in \mathbb{Z} / l \mathbb{Z}$, are exact.

b) For any $(C \rtimes G$ )-module $V$ over $R \# \Gamma$ which is projective over $R \# \Gamma$ (respectively over $R$ ) the modules $F_{a}(V), a \in \mathbb{Z} / l \mathbb{Z}$, are projective over $R \# \Gamma$ (respectively over $R$ ) again.

c) (Base change) The functors $F_{a}, a \in \mathbb{Z} / l \mathbb{Z}$, commute with base change with respect to any homomorphism $R \rightarrow R^{\prime}$ of $\Gamma$-rings.

Proof. This is clear.

\section{Example 1.}

a) For any $(C \rtimes G)$-module $V$ over $R \# \Gamma$ the module $F_{0}(V)$ is isomorphic to the fixed module $V^{c}:=\{v \in V: c(v)=v\}$. 
b) For any $a, b \in \mathbb{Z} / l \mathbb{Z} \backslash\{0\}$ the functors $F_{a}$ and $F_{b}$ are isomorphic.

c) Let $W$ be a $G$-module over $R \# \Gamma$. The canonical maps $C \rtimes G \rightarrow \operatorname{Permutations}(C)$ and $C \rtimes G \rightarrow G$ define an action of $C \rtimes G$ on the $R \# \Gamma$-module $\operatorname{Maps}(C, W)$. Then for each $a \in \mathbb{Z} / l \mathbb{Z}$ we have $F_{a}(\operatorname{Maps}(C, W)) \cong W$.

Proof. It easy to see that the isomorphism $V \rightarrow\left(S \otimes_{R} V\right)^{G}, v \mapsto \sum_{\sigma \in G} \zeta^{\sigma} \otimes \sigma(v)$, in the proof of Proposition 1 maps $V^{c}$ onto $F_{0}(V)$. This shows claim a). For claim b) we choose $\sigma \in G$ with $a=\sigma b$. Then $\left(S \otimes_{R} V\right)_{a} \rightarrow\left(S \otimes_{R} V\right)_{b}, \alpha \otimes v \mapsto \alpha \otimes \sigma(v)$ yields the desired isomorphism. The map

$$
S \otimes_{R} W \rightarrow\left(\operatorname{Maps}\left(C, S \otimes_{R} W\right)\right)_{a}, \quad x \mapsto\left(c^{i} \mapsto \zeta^{-i a} x\right),
$$

is a well-defined isomorphism of $G$-modules. Restricting to $G$-invariants yields the desired isomorphism in c).

The final assertions of this section will be based on the following Gauss sum argument.

Lemma 2. Let $S$ be a commutative ring such that $l$ is invertible in $S$ and such that $S$ contains a primitive $l$-th root of unity $\zeta$. Then the element $\sum_{\sigma \in G} \zeta^{\sigma}[\sigma]$ of the group ring $S G$ is invertible.

Proof. The following calculation shows that the element $\frac{1}{l} \sum_{\tau \in G}\left(\zeta^{-\tau}-1\right)\left[\tau^{-1}\right]$ is the inverse of $\sum_{\sigma \in G} \zeta^{\sigma}[\sigma]$.

$$
\left(\sum_{\sigma \in G} \zeta^{\sigma}[\sigma]\right) \cdot\left(\sum_{\tau \in G}\left(\zeta^{-\tau}-1\right)\left[\tau^{-1}\right]\right)=\sum_{\sigma, \tau \in G} \zeta^{\sigma}\left(\zeta^{-\tau}-1\right)\left[\sigma \tau^{-1}\right]=\sum_{\eta \in G}\left(\sum_{\tau \in G} \zeta^{\eta \tau}\left(\zeta^{-\tau}-1\right)\right)[\eta]=l[1] .
$$

Here the last equality follows from

$$
\sum_{\tau \in G} \zeta^{(\eta-1) \tau}-\sum_{\tau \in G} \zeta^{\eta \tau}=\left\{\begin{array}{ll}
(l-1)+1=l & \text { for } \eta=1 \\
0 & \text { for } \eta \in G \backslash\{1\}
\end{array} .\right.
$$

Proposition 2. If already $R$ contains a primitive $l$-th root of unity $\tilde{\zeta}$ then for each $a \in \mathbb{Z} / l \mathbb{Z}$ and for each $\left(C \rtimes G\right.$ )-module $V$ over $R \# \Gamma$ the canonical $R \# \Gamma$-linear map from $F_{a}(V)$ to the eigenspace $V_{a}:=\left\{v \in V: c(v)=\tilde{\zeta}^{a} v\right\}$ given by $\zeta \otimes v \mapsto \tilde{\zeta} v$ is an isomorphism.

Proof. The composition of the isomorphism $V \rightarrow\left(S \otimes_{R} V\right)^{G}, v \mapsto \sum_{\sigma \in G} \zeta^{\sigma} \otimes \sigma(v)$, used in the proof of Proposition 1 with the map $\left(S \otimes_{R} V\right)^{G} \rightarrow V, \zeta \otimes v \mapsto \tilde{\zeta} v$, is bijective by Lemma 2 . Hence the canonical map $\left(S \otimes_{R} V\right)^{G} \rightarrow V$ is an isomorphism and restricting to the eigenspaces yields the assertion.

Corollary. For any $(C \rtimes G$ )-module $V$ over $R \# \Gamma$ and for any $a \in \mathbb{Z} / l \mathbb{Z}$ the canonical map $S \otimes_{R} F_{a}(V) \rightarrow\left(S \otimes_{R} V\right)_{a}, \alpha \otimes x \mapsto \alpha x$, is an isomorphism.

Proof. Obviously, this map is the composition of the base change isomorphism $S \otimes_{R} F_{a}(V) \cong$ $F_{a}\left(S \otimes_{R} V\right)$ with the isomorphism $F_{a}\left(S \otimes_{R} V\right) \cong\left(S \otimes_{R} V\right)_{a}$ of Proposition 2.

The next example shows that our construction agrees with Kervaire's construction given in $\S 5$ of $[\mathrm{Ke}]$.

Example 2. Let $R=K$ be a field with $(\operatorname{char}(K), l)=1, L$ the splitting field of the polynomial $X^{l}-1$ over $K$ and $\tilde{\zeta} \in L$ a primitive $l$-th root of unity. Let $H$ be the Galois group of $L / K$ considered as a subgroup of $G$ as usual. As above for any $(C \times G)$-module $V$ over $K \# \Gamma$ we have a semilinear $H$-action on $L \otimes_{K} V$ and we let $\tilde{F}_{a}(V):=\left\{\sum_{\sigma \in H} \sigma(x): x \in\left(L \otimes_{K} V\right)_{a}\right\}$ be the image of the eigenspace $\left(L \otimes_{K} V\right)_{a}$ under the trace map. Then the canonical map $F_{a}(V) \rightarrow \tilde{F}_{a}(V)$ given by $\zeta \otimes v \mapsto \tilde{\zeta} \otimes v$ is an isomorphism. 
Proof. By the usual descent argument of Galois theory (e. g. see the appendix of [Dr]) we have $L \otimes_{K}\left(L \otimes_{K} V\right)_{a}^{H} \cong\left(L \otimes_{K} V\right)_{a}$ and hence $\tilde{F}_{a}(V)=\left(L \otimes_{K} V\right)_{a}^{H}$ because the trace map from $L$ to $K$ is surjective. By Proposition 2 and base change we furthermore have $\left(L \otimes_{K} V\right)_{a} \cong F_{a}\left(L \otimes_{K} V\right) \cong$ $L \otimes_{K} F_{a}(V)$. Now it easy to see that the composed isomorphism $L \otimes_{K} F_{a}(V) \cong L \otimes_{K}\left(L \otimes_{K} V\right)_{a}^{H}$ is the base change of the canonical map $F_{a}(V)=\left(S \otimes_{R} V\right)_{a}^{G} \rightarrow\left(L \otimes_{K} V\right)_{a}^{H}$ which therefore must be an isomorphism.

\section{Remark.}

a) Like in Example 2 one may use the $l$-th cyclotomic extension of $R$ (e. g. see page 34 of [Gre]) in place of $S=R[X] /\left(\Phi_{l}\right)$ for the construction of $F_{a}(V)$. However, one striking advantage of using $S=R[X] /\left(\Phi_{l}\right)$ is that we have the more or less canonical normal basis element $\zeta$ in $S$ and thus, for instance, base change becomes a very easy matter.

b) The quintessence of the above Gauss sum argument is the following assertion: For each $a \in \mathbb{Z} / l \mathbb{Z}$ the association $V \mapsto\left(\frac{1}{l} \sum_{\tau \in G}\left(\zeta^{-\tau}-1\right)\left[\tau^{-1}\right]\right) V_{a}$ is already defined over $\mathbb{Z}\left[\frac{1}{l}\right]$, i. e. for any $\left(C \rtimes G\right.$ )-module $V$ over $R \# \Gamma$ there exists a unique submodule $\tilde{V}_{a}$ of $V$ such that $S \otimes_{R} \tilde{V}_{a}=\frac{1}{l}\left(\sum_{\tau \in G}\left(\zeta^{-\tau}-1\right)\left[\tau^{-1}\right]\right)\left(\left(S \otimes_{R} V\right)_{a}\right)$ as submodules of $S \otimes_{R} V$.

\section{Adams Operations on $K_{0}(R \# \Gamma)$}

As in the previous section let $\Gamma$ be a group, $R$ a (noetherian) commutative ring and $\Gamma \times$ $R \rightarrow R$ an action of $\Gamma$ on $R$. In this section for each $k \in \mathbb{N}$ which is invertible in $R$ we will construct an Adams operation $\psi^{k}$ on the Grothendieck group $K_{0}(R \# \Gamma)$ of finitely generated, projective modules over the twisted group ring $R \# \Gamma$. Our construction generalizes the definition given by Kervaire in $\S 5$ of [Ke] which in turn is based on formula (2.7) of Atiyah's paper [At]. Furthermore, we will show that these Adams operations are multiplicative and that they commute with each other, with base change, and with the Cartan homomorphism from $K_{0}(R \# \Gamma)$ to the Grothendieck group $K_{0}(\Gamma, R)$ of finitely generated modules over $R \# \Gamma$ which are projective over $R$. Finally, if the characteristic of $R$ is a prime number, we will construct an Adams operation $\psi^{k}$ on $K_{0}(R \# \Gamma)$ for an arbitrary $k \in \mathbb{N}$.

In general there do not exist exterior power operations on $K_{0}(R \# \Gamma)$ which are compatible with the Cartan homomorphism (see Remarque on page 19 of [Ke]). But we still have tensor products on $K_{0}(R \# \Gamma)$ by the following lemma.

Lemma 3. Let $W$ be a projective $R \# \Gamma$-module and $V$ an $R \# \Gamma$-module such that there is an $R \# \Gamma$-module $V^{\prime}$ with $V \oplus V^{\prime}$ free over $R$. (For instance, this assumption is satisfied if $V$ is even free over $R$ or if $V$ is $R \# \Gamma$-projective or if $V$ is projective over $R$ and the action of $\Gamma$ on $R$ is trivial.) Then the $R \# \Gamma$-module $V \otimes_{R} W$ (with diagonal $\Gamma$-action) is projective over $R \# \Gamma$.

Proof. We may assume that $W=R \# \Gamma$ and that $V$ is $R$-free, say with basis $x_{i}, i \in I$. Then $x_{i} \otimes 1, i \in I$, is a basis of $V \otimes W$ over $R \# \Gamma$ : The $i$-th dual basis element $\left(x_{i} \otimes 1\right)^{*}$ is given by $x \otimes[\gamma] \mapsto x_{i}^{*}\left(\left[\gamma^{-1}\right] \cdot x\right)[\gamma]$.

Now let $l$ be a prime number which is invertible in $R$ and $V$ an $R \# \Gamma$-module. The symmetric group $\Sigma_{l}:=$ Permutations $(\mathbb{Z} / l \mathbb{Z})$ acts on the $R \# \Gamma$-module $V^{\otimes l}:=\otimes_{i \in \mathbb{Z} / l \mathbb{Z}} V$ by $\sigma\left(\otimes_{i} v_{i}\right):=$ $\otimes_{i} v_{\sigma^{-1}(i)}$ for $\sigma \in \Sigma_{l}, v_{i} \in V$. We consider the semidirect product $C \rtimes G$ defined in section 1 as a subgroup of $\Sigma_{l}$ by mapping the generator $c$ of $C$ to the cycle $\mathbb{Z} / l \mathbb{Z} \rightarrow \mathbb{Z} / l \mathbb{Z}, i \mapsto i+1$, and by embedding $G$ in $\Sigma_{l}$ via $G=(\mathbb{Z} / l \mathbb{Z})^{\times}=\operatorname{Aut}(\mathbb{Z} / l \mathbb{Z}) \subseteq$ Permutations $(\mathbb{Z} / l \mathbb{Z})=\Sigma_{l}$. So $V^{\otimes l}$ becomes a $(C \rtimes G)$-module over $R \# \Gamma$. 


\section{Definition.}

a) For any $a \in \mathbb{Z} / l \mathbb{Z}$ the $R \# \Gamma$-module

$$
V[a]:=V[a]_{l}:=F_{a}\left(V^{\otimes l}\right)
$$

is called the cyclic $l$-th power of $V$ with eigenvalue $\zeta_{l}^{a}$.

b) For any finitely generated, projective $R \# \Gamma$-module $V$ we let

$$
\psi^{l}(V):=V[0]-V[1] \in K_{0}(R \# \Gamma) .
$$

c) For any finitely generated $R \# \Gamma$-module $V$ which is projective over $R$ we let

$$
\hat{\psi}^{l}(V):=V[0]-V[1] \in K_{0}(\Gamma, R) .
$$

Remark. In [At] Atiyah gives the name "cyclic $l$-th power" to a different but related object. The expression "with eigenvalue $\zeta_{l}^{a}$ " in the above definition is only a name and actually has not a real meaning. The definitions b) and c) make sense because of Lemma 3 and Corollary b) of Proposition 1.

Example 3. For any $R \# \Gamma$-module $V$ which is projective of rank 1 over $R$ we have $\hat{\psi}_{l}(V)=$ $V^{\otimes l}$ in $K_{0}(\Gamma, R)$.

Proof. The symmetric group and in particular $C$ act on $V^{\otimes l}$ trivially. Hence $V[0]=F_{0}\left(V^{\otimes l}\right) \cong$ $V^{\otimes l}$ by Example 1a) and $V[1]=0$ by Proposition 1 .

Proposition 3. Let $0 \rightarrow V_{0} \rightarrow V_{1} \rightarrow V_{2} \rightarrow 0$ be an exact sequence of finitely generated, projective $R \# \Gamma$-modules. Then $\psi^{l}\left(V_{1}\right)=\psi^{l}\left(V_{0}\right)+\psi^{l}\left(V_{2}\right)$ in $K_{0}(R \# \Gamma)$. The analogous assertion holds for $\hat{\psi}^{l}$ on $K_{0}(\Gamma, R)$.

Proof. We recall the essential steps of Kervaire's proof (see $\S 5$ of [Ke]) for the reader's convenience since this proof motivates the construction of Adams operations on higher $K$-groups given in the next section and since some part of this proof will be referred to in the proof of the Propositions 6 and 8 .

We consider the following filtration on $V_{1}^{\otimes l}$. For $\varepsilon \in\{0,1\}^{\mathbb{Z} / l \mathbb{Z}}$ let $|\varepsilon|:=\sum_{i \in \mathbb{Z} / l \mathbb{Z}} \varepsilon_{i}, V_{\varepsilon}:=$ $\otimes_{i} V_{\varepsilon_{i}}$ and $W_{\varepsilon}:=\otimes_{i} V_{\delta_{i}}$ with $\delta_{i}=0$ if $\varepsilon_{i}=0$ and $\delta_{i}=2$ if $\varepsilon_{i}=1$. Now for $\lambda=0, \ldots, l$ let $Q_{\lambda}:=\sum_{|\varepsilon|=\lambda} V_{\varepsilon}$. Then obviously

$$
0 \subset Q_{0}=V_{0}^{\otimes l} \subset Q_{1} \subset \ldots \subset Q_{l}=V_{1}^{\otimes l}
$$

is a filtration of $V_{1}^{\otimes l}$ by $(C \rtimes G)$-submodules over $R \# \Gamma$. For each $\lambda=0, \ldots, l$ the canonical surjections $V_{\varepsilon} \rightarrow W_{\varepsilon}$ induce a $(C \rtimes G)$-isomorphism of the successive quotient $Q_{\lambda} / Q_{\lambda-1}$ with $\oplus_{|\varepsilon|=\lambda} W_{\varepsilon}$. (This fact has been implicitly proved in sections 2 and 3 of [Ko2] for the construction of shuffle products on higher $K$-theory. It should be regarded as an analogue of the fact that the successive quotients of the (Koszul) filtration of the exterior power $\Lambda^{l}\left(V_{1}\right)$ by the submodules $V_{0} \wedge \ldots \wedge V_{0} \wedge V_{1} \wedge \ldots \wedge V_{1}$ are isomorphic to $\Lambda^{l-\lambda} V_{0} \otimes \Lambda^{\lambda} V_{2}, \lambda=0, \ldots, l$.) Hence the successive quotients are projective $R \# \Gamma$-modules by Lemma 3 and for each $a \in \mathbb{Z} / l \mathbb{Z}$ we have

$$
F_{a}\left(V_{1}^{\otimes l}\right)=F_{a}\left(V_{0}^{\otimes l}\right)+F_{a}\left(V_{2}^{\otimes l}\right)+\sum_{\lambda=1}^{l-1} F_{a}\left(Q_{\lambda} / Q_{\lambda-1}\right) \quad \text { in } \quad K_{0}(R \# \Gamma)
$$

by Corollary a) of Proposition 1. Now, in order to prove Proposition 3 it suffices to show that for each $\lambda \neq 0, l$ the module $F_{a}\left(Q_{\lambda} / Q_{\lambda-1}\right)$ is independent of $a \in \mathbb{Z} / l \mathbb{Z}$. 
By the succeeding lemma there is a $G$-stable system of representatives $R_{\lambda}$ in $M_{\lambda}:=\{\varepsilon \in$ $\left.\{0,1\}^{\mathbb{Z} / l \mathbb{Z}}:|\varepsilon|=\lambda\right\}$ for the natural action of $C$ on $M_{\lambda}$. Obviously, for $\lambda \neq 0, l$ there are no fixed points for this action of $C$ on $M_{\lambda}$. Hence $\oplus_{|\varepsilon|=\lambda} W_{\varepsilon}$ may be identified with the $(C \rtimes G)$ module $\operatorname{Maps}\left(\mathbb{Z} / l \mathbb{Z}, \oplus_{\varepsilon \in R_{\lambda}} W_{\lambda}\right)$ where $C \rtimes G$ acts on $\mathbb{Z} / l \mathbb{Z}$ via the embedding $C \rtimes G \subset \Sigma_{l}$ and on $\oplus_{\varepsilon \in R_{\lambda}} W_{\varepsilon}$ via the canonical surjection $C \rtimes G \rightarrow G$. By Example 1c) the $R \# \Gamma$-module $F_{a}\left(Q_{\lambda} / Q_{\lambda-1}\right)$ is isomorphic to $\oplus_{\varepsilon \in R_{\lambda}} W_{\varepsilon}$ for each $a \in \mathbb{Z} / l \mathbb{Z}$ and in particular it is independent of $a \in \mathbb{Z} / l \mathbb{Z}$. This ends the proof of the proposition for $K_{0}(R \# \Gamma)$. For $K_{0}(\Gamma, R)$ the same proof works.

Lemma 4. For each $(C \rtimes G)$-set $M$ there exists a $G$-stable system of representatives in $M$ for the set of $C$-orbits $N:=M / C$.

Proof. We may assume that the induced action of $G$ on $N$ is transitive. Let $I \subseteq G$ be the isotropy group of some $n \in N$. It suffices to show that there is a preimage of $n$ in $M$ which is fixed by $I$. Let $m \in M$ be any preimage of $n$ and $\sigma$ a generator of $I$. There is some $a \in \mathbb{Z} / l \mathbb{Z}$ such that $\sigma m=c^{a} m$. Then $c^{b} m$ with $b=0$ if $\sigma=1$ and $b=(1-\sigma)^{-1} a$ if $\sigma \neq 1$ is an $I$-fixed preimage of $n$.

By Proposition 3 the association $V \mapsto \psi^{l}(V)$ extends to a well-defined homomorphism

$$
\psi^{l}: K_{0}(R \# \Gamma) \rightarrow K_{0}(R \# \Gamma) .
$$

Definition. The homomorphism $\psi^{l}$ is called the $l$-th Adams operation on $K_{0}(R \# \Gamma)$.

\section{Remark.}

a) In the next section we will show that for any $a \in \mathbb{Z} / l \mathbb{Z}$ already the association $V \mapsto V[a]_{l}$ extends to a well-defined map on $K_{0}(R \# \Gamma)$.

b) Let $R=K$ be a field with $(\operatorname{char}(K), l)=1$, let $\Gamma$ be a finite group and let $\Gamma$ act on $R$ trivially. Then Example 2 shows that the definition of the $l$-th Adams operation given here agrees with Kervaire's definition in $\S 5$ of $[\mathrm{Ke}]$.

c) Let $f: R \rightarrow R^{\prime}$ be an homomorphism of $\Gamma$-rings. Then Corollary c) of Proposition 1 shows that the Adams operation $\psi^{l}$ commutes with the base change homomorphism $f^{*}: K_{0}(R \# \Gamma) \rightarrow$ $K_{0}\left(R^{\prime} \# \Gamma\right)$ given by $V \mapsto R^{\prime} \otimes_{R} V$.

d) Obviously, $\psi^{l}$ commutes with the restricting homomorphism $K_{0}(R \# \Gamma) \rightarrow K_{0}\left(R \# \Gamma^{\prime}\right)$ with respect to any homomorphism of groups $\Gamma^{\prime} \rightarrow \Gamma$.

By Proposition 3 the association $V \mapsto \hat{\psi}^{l}(V)$ induces a well-defined homomorphism

$$
\hat{\psi}^{l}: K_{0}(\Gamma, R) \rightarrow K_{0}(\Gamma, R) .
$$

By section (2.5) of [Ko1] the exterior power operations give $K_{0}(\Gamma, R)$ a (special) $\lambda$-ring structure. Thus we can define an Adams operation $\psi^{l}$ on $K_{0}(\Gamma, R)$ via the $l$-th Newton polynomial (e. g. see chapter I, $\S 6$ of $[\mathrm{FL}])$.

Proposition 4. The $l$-th Adams operation $\psi^{l}$ agrees with the homomorphism $\hat{\psi}^{l}$ on $K_{0}(\Gamma, R)$. In particular, the Cartan homomorphism

$$
c: K_{0}(R \# \Gamma) \rightarrow K_{0}(\Gamma, R)
$$

commutes with the $l$-th Adams operation.

Proof. Let $V$ be a finitely generated, $R$-projective $R \# \Gamma$-module, say of rank $r$. We have to show that $\psi^{l}(V)=\hat{\psi}^{l}(V)$ in $K_{0}(\Gamma, R)$. By the geometric splitting principle (see section (2.5) of [Ko1]) there is a $\Gamma$-scheme $f: X \rightarrow \operatorname{Spec}(R)$ such that the base change homomorphism 
$f^{*}: K_{0}(\Gamma, R) \rightarrow K_{0}(\Gamma, X)$ is injective and such that we have $f^{*}(V)=\mathcal{L}_{1}+\ldots+\mathcal{L}_{r}$ with $\Gamma$ modules $\mathcal{L}_{1}, \ldots, \mathcal{L}_{r}$ on $X$ of rank 1. (Even if the action of $\Gamma$ on $R$ is trivial the action of $\Gamma$ on $X$ usually is not trivial. This is the essential reason for considering not only usual group rings but twisted group rings in this paper.) Similar to the affine case for any locally free $\Gamma$-module $\mathcal{V}$ on $X$ and for any $a \in \mathbb{Z} / l \mathbb{Z}$ we let $\mathcal{V}[a]:=p_{*}\left(\left(p^{*} \mathcal{V}^{\otimes l}\right)_{a}^{G}\right)$, where $p: S \otimes_{R} X \rightarrow X$ is the canonical projection, and we let $\hat{\psi}^{l}(\mathcal{V}):=\mathcal{V}[0]-\mathcal{V}[1] \in K_{0}(\Gamma, X)$. The same proofs as above show that the association $\mathcal{V} \mapsto \hat{\psi}^{l}(\mathcal{V})$ induces a well-defined homomorphism $\hat{\psi}^{l}$ on $K_{0}(\Gamma, X)$ and that $\hat{\psi}^{l}$ commutes with the base change homomorphism $f^{*}: K_{0}(\Gamma, R) \rightarrow K_{0}(\Gamma, X)$. Now an analogue of Example 3 for $X$ shows the proposition.

By Lemma 3 we have a ring structure on $K_{0}(R \# \Gamma)$ (without 1 in general). If the action of $\Gamma$ on $R$ is trivial, again by Lemma 3 we have a $K_{0}(\Gamma, R)$-module structure on $K_{0}(R \# \Gamma)$.

Proposition 5. The $l$-th Adams operation $\psi^{l}: K_{0}(R \# \Gamma) \rightarrow K_{0}(R \# \Gamma)$ is multiplicative. If the action of $\Gamma$ on $R$ is trivial, $\psi^{l}$ is semilinear with respect to the $l$-th Adams operation $\psi^{l}$ on $K_{0}(\Gamma, R)$.

Proof. By the Corollary of Proposition 2 for any $(C \times \mid G)$-module $V$ over $R \# \Gamma$ the canonical map $S \otimes_{R}\left(S \otimes_{R} V\right)^{G} \rightarrow S \otimes_{R} V$ is an isomorphism. Hence for any $(C \rtimes G)$-modules $V, W$ over $R \# \Gamma$ the canonical map

$$
\left(S \otimes_{R} V\right)^{G} \otimes_{R}\left(S \otimes_{R} W\right)^{G} \rightarrow\left(S \otimes_{R} V \otimes_{R} W\right)^{G}, \quad(\alpha \otimes v) \otimes(\beta \otimes w) \mapsto \alpha \beta \otimes v \otimes w
$$

becomes an isomorphism after tensoring with $S$ over $R$ and thus this map itself is an isomorphism. Restricting to the eigenspaces yields an isomorphism $\oplus_{a+b=c} F_{a}(V) \otimes_{R} F_{b}(W) \cong$ $F_{c}\left(V \otimes_{R} W\right)$ for each $c \in \mathbb{Z} / l \mathbb{Z}$. Hence using Example 1b) we get the following equality in $K_{0}(R \# \Gamma)$ for finitely generated, projective $R \# \Gamma$-modules $V, W$ :

$$
\begin{aligned}
\psi^{l}(V & \otimes W)=\sum_{a \in \mathbb{Z} / l \mathbb{Z}} V[a] \otimes W[-a]-\sum_{a \in \mathbb{Z} / l \mathbb{Z}} V[a] \otimes W[1-a] \\
& =(V[0] \otimes W[0]+(l-1) V[1] \otimes W[1])-(V[0] \otimes W[1]+V[1] \otimes W[0]+(l-2) V[1] \otimes W[1]) \\
& =(V[0]-V[1]) \cdot(W[0]-W[1])=\psi^{l}(V) \cdot \psi^{l}(W) .
\end{aligned}
$$

This shows the first assertion of Proposition 5. The same proof shows the second assertion.

Proposition 6. Let $k \in \mathbb{N}$ be another prime number which is invertible in $R$. Then the Adams operations $\psi^{k}$ and $\psi^{l}$ on $K_{0}(R \# \Gamma)$ commute.

Proof. Here let $C$ be the cyclic group of order $k l, c$ a generator of $C, G:=(\mathbb{Z} / k l \mathbb{Z})^{\times}$ acting on $C, \Phi_{k l}$ the $k l$-th cyclotomic polynomial, $S:=R[X] /\left(\Phi_{k l}\right)$ and $\zeta:=\bar{X} \in S$. We will denote the corresponding objects for $k$ respectively $l$ with a subscript $k$ respectively $l$. We have canonical isomorphisms

$$
\mathbb{Z} / k l \mathbb{Z} \stackrel{\sim}{\rightarrow} \mathbb{Z} / k \mathbb{Z} \times \mathbb{Z} / l \mathbb{Z} \quad \text { and } \quad S \stackrel{\sim}{\rightarrow} S_{k} \otimes_{R} S_{l}, \zeta \mapsto \zeta_{k} \otimes \zeta_{l}
$$

and for any $a \in \mathbb{Z} / k l \mathbb{Z}$ we will denote both the element $a+k \mathbb{Z}$ in $\mathbb{Z} / k \mathbb{Z}$ and the preimage of $(a+k \mathbb{Z}, 0)$ in $\mathbb{Z} / k l \mathbb{Z}$ by $a_{k}$.

Now let $V$ be a projective $R \# \Gamma$-module. As above for any $a \in \mathbb{Z} / k l \mathbb{Z}$ let

$$
V[a]:=\left(S \otimes_{R} V^{\otimes k l}\right)_{a}^{G} .
$$

We claim that

$$
\left.V[0]-V\left[1_{k}\right] \stackrel{\text { def }}{=} V[(0,0)]-V[(0,1)]\right)=V[0]_{k}[0]_{l}-V[0]_{k}[1]_{l} \quad \text { in } \quad K_{0}(R \# \Gamma),
$$




$$
V\left[1_{l}\right]-V[1](\stackrel{\text { def }}{=} V[(1,0)]-V[(1,1)])=V[1]_{k}[0]_{l}-V[1]_{k}[1]_{l} \quad \text { in } \quad K_{0}(R \# \Gamma) .
$$

Then we have

$$
\psi^{l} \circ \psi^{k}(V)=V[0]-V\left[1_{l}\right]-V\left[1_{k}\right]+V[1] \quad \text { in } \quad K_{0}(R \# \Gamma)
$$

and this expression is symmetric in $k$ and $l$. Thus these claims show the proposition.

To prove the above claims we proceed like in the proof of Proposition 5. Using Lemma 2 for $k$ and $l$ one shows like in the proof of the Corollary of Proposition 2 that the canonical map

$$
S \otimes_{R}\left(S \otimes_{R} V^{\otimes k l}\right)^{G} \rightarrow S \otimes_{R} V^{\otimes k l}, \quad \alpha \otimes x \mapsto \alpha x,
$$

is an isomorphism. Hence after tensoring with $S=S_{k} \otimes S_{l}$ the map

$$
\Phi:\left(S_{l} \otimes_{R}\left(\left(S_{k} \otimes_{R} V^{\otimes k}\right)^{G_{k}}\right)^{\otimes l}\right)^{G_{l}} \rightarrow\left(S \otimes_{R} V^{\otimes k l}\right)^{G}
$$

induced by the canonical isomorphism $\left(V^{\otimes k}\right)^{\otimes l} \cong V^{\otimes k l}$ becomes an isomorphism and thus already $\Phi$ itself is an isomorphism.

Now we consider the eigenspace decompositions on both sides of $\Phi$. By Lemma 4 there exists a $G$-stable system of representatives $R$ in $(\mathbb{Z} / k \mathbb{Z})^{\mathbb{Z} / l \mathbb{Z}}$ for the set of $C$-orbits under the natural action of $C$ on $(\mathbb{Z} / k \mathbb{Z})^{\mathbb{Z} / l \mathbb{Z}} \backslash\{(a, \ldots, a): a \in \mathbb{Z} / k \mathbb{Z}\}$. Like in the proof of Proposition 3 this action has no fixed points and we have

$$
\begin{aligned}
\left(\left(S_{k}\right.\right. & \left.\left.\otimes V^{\otimes k}\right)^{G_{k}}\right)^{\otimes l} \cong\left(V[0]_{k} \oplus \ldots \oplus V[k-1]_{k}\right)^{\otimes l} \\
& \cong \underset{\varepsilon \in(\mathbb{Z} / k \mathbb{Z})^{\mathbb{Z} l \mathbb{Z}}}{\oplus} V\left[\varepsilon_{0}\right]_{k} \otimes \ldots \otimes V\left[\varepsilon_{l-1}\right]_{k} \\
& \cong \bigoplus_{a=0}^{k-1} V[a]_{k}^{\otimes l} \oplus \underset{\varepsilon \in R}{\oplus} \operatorname{Maps}\left(\mathbb{Z} / l \mathbb{Z}, V\left[\varepsilon_{0}\right]_{k} \otimes \ldots \otimes V\left[\varepsilon_{l-1}\right]_{k}\right) .
\end{aligned}
$$

Thus for each $b \in \mathbb{Z} / l \mathbb{Z}$ we have

$$
\left(\left(S_{k} \otimes V^{\otimes k}\right)^{G_{k}}\right)[b]_{l} \cong \underset{a=0}{\oplus_{a=0}^{-1}} V[a]_{k}[b]_{l} \oplus \underset{\varepsilon \in R}{\oplus} V\left[\varepsilon_{0}\right]_{k} \otimes \ldots \otimes V\left[\varepsilon_{l-1}\right]_{k}
$$

by Example 1c). For any $\varepsilon \in(\mathbb{Z} / k \mathbb{Z})^{\mathbb{Z} / l \mathbb{Z}}$ let $|\varepsilon|:=\sum_{i=0}^{l-1} \varepsilon_{i} \in \mathbb{Z} / k \mathbb{Z}$. Then for each $(a, b) \in \mathbb{Z} / k \mathbb{Z} \times \mathbb{Z} / l \mathbb{Z}=\mathbb{Z} / k l \mathbb{Z}$ we have

$$
\Phi^{-1}(V[(a, b)]) \cong V\left[l^{-1} a\right]_{k}[b]_{l} \oplus \underset{\varepsilon \in R:|\varepsilon|=a}{\oplus} V\left[\varepsilon_{0}\right]_{k} \otimes \ldots \otimes V\left[\varepsilon_{l-1}\right]_{k} .
$$

Because of Example 1b) these isomorphisms show the above claims and Proposition 6 is proved.

\section{Remark.}

a) Let $k \in \mathbb{N}$ be invertible in $R$ and let $k=l_{1} \cdot \ldots \cdot l_{r}$ be its prime factor decomposition. Then by Proposition 6 the composition $\psi^{k}:=\psi^{l_{1}} \circ \ldots \circ \psi^{l_{r}}$ does not depend on the order of the prime numbers $l_{1}, \ldots, l_{r}$. We call this composition the $k$-th Adams operation on $K_{0}(R \# \Gamma)$. Obviously, we have $\psi^{k} \circ \psi^{l}=\psi^{k l}$ for all $k, l \in \mathbb{N}$ which are invertible in $R$. Like in the proof of Proposition 6 one can prove the following formula for $\psi^{k}(V), V$ a finitely generated $R \# \Gamma$-module:

$$
\psi^{k}(V)=\sum_{d \mid k} \mu(d) F_{d}\left(V^{\otimes k}\right) \quad \text { in } \quad K_{0}(R \# \Gamma)
$$

(cf. Proposition 2 of [Be]). Here $\mu$ is the Möbius function of multiplicative number theory and $F_{d}\left(V^{\otimes k}\right)$ is the analogously defined cyclic $k$-th power of $V$ with eigenvalue $\zeta_{k}^{k / d}$. 
b) In the situation considered by Kervaire in [Ke] (cf. Remark b) before Proposition 4) the Cartan homomorphism $c$ is injective and the assertions of the Propositions 5 and 6 already follow from the corresponding assertions for $K_{0}(\Gamma, R)$.

In the final part of this section we assume in addition that the characteristic of $R$ is a prime number $p$. Let

$$
F: R \rightarrow R, \quad r \mapsto r^{p},
$$

be the Frobenius endomorphism. Obviously, $F$ is a homomorphism of $\Gamma$-rings. Thus we have the base change homomorphism

$$
F^{*}: K_{0}(R \# \Gamma) \rightarrow K_{0}(R \# \Gamma), \quad V \mapsto R \otimes_{R} V,
$$

where the tensor product $R \otimes_{R} V$ is taken with respect to $F$ and the $R$-module structure of $R \otimes_{R} V$ is given by $r(s \otimes v):=r s \otimes v$ for $r, s \in R, v \in V$ as usual.

Definition. The base change homomorphism $F^{*}$ is called the $p$-th Adams operation on $K_{0}(R \# \Gamma)$ and will be denoted by $\psi^{p}$.

Remark. As already shown $F^{*}=\psi^{p}$ commutes with $\psi^{l}$ for each prime number $l$. Thus here we can define an Adams operation $\psi^{k}$ for an arbitrary $k \in \mathbb{N}$.

Proposition 7. The base change homomorphism $F^{*}$ on $K_{0}(\Gamma, R)$ agrees with the $p$-th Adams operation $\psi^{p}$ on $K_{0}(\Gamma, R)$. In particular, $\psi^{p}$ commutes with the Cartan homomorphism $c$ : $K_{0}(R \# \Gamma) \rightarrow K_{0}(\Gamma, R)$.

Proof. Let $V$ be a finitely generated $R \# \Gamma$-module which is projective over $R$. We have to show that $\psi^{p}(V)=F^{*}(V)$ in $K_{0}(\Gamma, R)$. Like in the proof of Proposition 4 by the geometric splitting principle we may assume that $V$ is of rank 1 . Then

$$
F^{*}(V)=R \otimes_{R} V \rightarrow V^{\otimes p}, \quad r \otimes v \mapsto r \cdot v^{\otimes p},
$$

is a well-defined isomorphism of $R \# \Gamma$-modules. This shows Proposition 7 .

Question. Let $p$ be a prime number which is neither invertible nor zero in $R$. Is there an Adams operation $\psi^{p}$ on $K_{0}(R \# \Gamma)$ which commutes both with the base change homomorphism $K_{0}(R \# \Gamma) \rightarrow K_{0}(R /(p) \# \Gamma)$ and with the base change homomorphism $K_{0}(R \# \Gamma) \rightarrow$ $K_{0}\left(R\left[\frac{1}{p}\right] \# \Gamma\right) ?$

\section{Cyclic Powers and Adams Operations on $K_{q}(R \# \Gamma)$}

Again let $\Gamma$ be a group, $R$ a commutative $\Gamma$-ring and $l$ a prime number which is invertible in $R$. For any $q \geq 0$ let $K_{q}(R \# \Gamma)$ be Quillen's $q$-th $K$-group associated with the exact category $\mathcal{M}$ of finitely generated, projective $R \# \Gamma$-modules. In this section we will construct cyclic power operations $[a]_{l}, a \in \mathbb{Z} / l \mathbb{Z}$, and an Adams operation $\psi^{l}$ on $K_{q}(R \# \Gamma)$. This construction uses shuffle products in higher $K$-theory (cf. [Ko2]) and Grayson's techniques of constructing exterior power operations on higher $K$-theory (cf. [Gr1]). We will show that for $q=0$ this construction agrees with the construction given in section 2. Furthermore we will give some speculations how one should be able to prove that $\psi^{l}$ commutes with the Cartan homomorphism $c: K_{q}(R \# \Gamma) \rightarrow K_{q}(\Gamma, R)$.

At first we will recall some definitions of [Gr1] and [Ko2]; in particular we will give a definition of Quillen's $q$-th $K$-group $K_{q}(R \# \Gamma)$. Let Ord be the category of finite, non-empty, (partially) 
ordered sets. For any $A \in$ Ord let the disjoint union $\{L, R\} \cup A$ be ordered in such a way that $A$ is an ordered subset, that $L<a$ and $R<a$ for all $a \in A$ and that $L$ and $R$ are not comparable. Let $\Gamma(A):=\left\{\frac{i}{j}: i \in A, j \in\{L, R\} \cup A, j \leq i\right\}$ and more generally let $\Gamma^{l}(A):=$ $\left\{\left(\frac{i_{1}}{j_{1}}, *_{2}, \ldots, *_{l}, \frac{i_{l}}{j_{l}}\right):\right.$ for all $r=1, \ldots, k: i_{r} \in A, j_{r} \in\{L, R\} \cup A, j_{r} \leq i_{r}$; for all $r=2, \ldots, k$ : $*_{r} \in\{\wedge, \otimes\}$ and, if $*_{r}=\wedge$, then $j_{r-1}=j_{r}$ and $\left.i_{r-1} \leq i_{r}\right\}$. In section 5 of [Gr1] Grayson defines a certain (partial) order on $\Gamma(A)$ and $\Gamma^{l}(A)$ and calls certain sequences $\alpha^{\prime} \rightarrow \alpha \rightarrow \alpha^{\prime \prime}$ in $\Gamma(A)$ and $\Gamma^{l}(A)$ (short) exact sequences. Then the so-called $G$-construction is the simplicial set

$$
G \mathcal{M}: \operatorname{Ord}^{\text {op }} \rightarrow \text { Ens, } \quad A \mapsto \operatorname{Exact}(\Gamma(A), \mathcal{M}),
$$

where $\operatorname{Exact}(\Gamma(A), \mathcal{M})$ denotes the set of all functors of $\Gamma(A)$ to $\mathcal{M}$ which transform short exact sequences in $\Gamma(A)$ to short exact sequences of $\mathcal{M}$. By [GG] the $q$-th homotopy group $\pi_{q}(|G \mathcal{M}|)$ of the geometric realization $|G \mathcal{M}|$ of $G \mathcal{M}$ is Quillen's $q$-th $K$-group $K_{q}(R \# \Gamma)$. Similarly let $\mathcal{H}^{l} \mathcal{M}$ be the simplicial set

$$
\mathcal{H}^{l} \mathcal{M}: \operatorname{Ord}^{\text {op }} \rightarrow \text { Ens, } \quad A \mapsto \operatorname{Exact}\left(\Gamma^{l}(A), \mathcal{M}\right) .
$$

By Lemma 3 we have an associative, commutative and bi-exact tensor product $\otimes: \mathcal{M} \times \mathcal{M} \rightarrow$ $\mathcal{M}$. Associated with this functor we have a simplicial map

$$
\otimes: \underbrace{G \mathcal{M} \times \ldots \times G \mathcal{M}}_{l} \rightarrow \mathcal{H}^{l} \mathcal{M}
$$

the so-called $(1, \ldots, 1)$-shuffle product (cf. [Ko2]). It is defined as follows: For any $A \in$ Ord and any $\alpha=\left(\frac{i_{1}}{j_{1}}, *_{2}, \ldots, *_{l}, \frac{i_{l}}{j_{l}}\right) \in \Gamma^{l}(A)$ let $\sim_{\alpha}$ be the equivalence relation on $\Sigma_{l}:=$ Permutations $\{1, \ldots, l\}$ defined by $\sigma \sim_{\alpha} \tau$, iff for all $r=1, \ldots, l$ there are only $\wedge$ 's in $\alpha$ between the places $\sigma(r)$ and $\tau(r)$. Then on the level $A$ the simplical map $\otimes$ is given by

$$
\begin{aligned}
G \mathcal{M}(A) \times \ldots \times G \mathcal{M}(A) & \rightarrow \mathcal{H}^{l} \mathcal{M}(A)=\operatorname{Exact}\left(\Gamma^{l}(A), \mathcal{M}\right) \\
\left(M_{1}, \ldots, M_{l}\right) & \mapsto\left(\alpha \mapsto \underset{\tilde{\sigma} \in \Sigma_{l} / \sim_{\alpha}}{\bigoplus} \sum_{\sigma \in \tilde{\sigma}} M_{1}\left(\frac{i_{\sigma(1)}}{j_{\sigma(1)}}\right) \otimes \ldots \otimes M_{l}\left(\frac{i_{\sigma(l)}}{j_{\sigma(l)}}\right)\right)
\end{aligned}
$$

(cf. Remark at the end of section 3 of [Ko2]). We call the composition

$$
{ }^{\otimes l}: G \mathcal{M} \stackrel{\Delta}{\rightarrow} G \mathcal{M} \times \ldots \times G \mathcal{M} \stackrel{\otimes}{\rightarrow} \mathcal{H}^{l} \mathcal{M}
$$

of the diagonal $\Delta$ with the $(1, \ldots, 1)$-shuffle product $\otimes$ the $l$-th shuffle power.

Next for any $A \in$ Ord and any $M \in G \mathcal{M}(A)$ we will define a natural action of $\Sigma_{l}$ on $M^{\otimes l} \in \operatorname{Exact}\left(\Gamma^{l}(A), \mathcal{M}\right)=\mathcal{H}^{l} \mathcal{M}(A)$. Let $\alpha=\left(\frac{i_{1}}{j_{1}}, *_{2}, \ldots, *_{l}, \frac{i_{l}}{j_{l}}\right) \in \Gamma^{l}(A)$. Then interchanging the direct summands yields an action of $\Sigma_{l}$ on $\oplus_{\sigma \in \Sigma_{l}} M\left(\frac{i_{\sigma(1)}}{j_{\sigma(1)}}\right) \otimes \ldots \otimes M\left(\frac{i_{\sigma(l)}}{j_{\sigma(l)}}\right)$. Obviously for any $\sigma_{1}, \sigma_{2} \in \Sigma_{l}$ with $\sigma_{1} \sim_{\alpha} \sigma_{2}$ we have $\sigma_{1} \circ \tau \sim_{\alpha} \sigma_{2} \circ \tau$ again for all $\tau \in \Sigma_{l}$. Hence by Lemma 2.1a) of [Ko2] this action of $\Sigma_{l}$ induces an action of $\Sigma_{l}$ on $M^{\otimes l}(\alpha)=\oplus_{\tilde{\sigma} \in \Sigma_{l} / \sim_{\alpha}} \sum_{\sigma \in \tilde{\sigma}} M\left(\frac{i_{\sigma(1)}}{j_{\sigma(1)}}\right) \otimes \ldots \otimes M\left(\frac{i_{\sigma(l)}}{j_{\sigma(l)}}\right)$. Finally for any $\alpha, \beta \in \Gamma^{l}(A)$ with $\alpha \rightarrow \beta$ the corresponding map $M^{\otimes l}(\alpha) \rightarrow M^{\otimes l}(\beta)$ is compatible with this $\Sigma_{l}$-action. Thus $M^{\otimes l}$ may be viewed as an element of $\operatorname{Exact}\left(\Gamma^{l}(A), \mathcal{M}^{\Sigma_{l}}\right)$; here $\mathcal{M}^{\Sigma_{l}}$ denotes the category of $\Sigma_{l}$-modules over $R \# \Gamma$ which are finitely generated and projective over $R \# \Gamma$.

Remark. Let $\Gamma=\{1\}$ be the trivial group. In the last example of [Gr1] Grayson considers the operations

$$
\mathcal{M}^{\Sigma_{n}} \times \mathcal{M}^{\Sigma_{p}} \rightarrow \mathcal{M}^{\Sigma_{n+p}}, \quad(V, W) \mapsto \operatorname{Ind}_{\Sigma_{n} \times \Sigma_{p}}^{\Sigma_{n+p}} V \otimes_{R} W
$$


and

$$
F_{k}\left(\mathcal{M}^{\Sigma_{n}}\right) \rightarrow \mathcal{M}^{\Sigma_{n k}}, \quad\left(V_{1} \hookrightarrow \ldots \hookrightarrow V_{k}\right) \mapsto \operatorname{Ind}_{\Sigma_{n} \int \Sigma_{k}}^{\Sigma_{n k}} \sum_{\sigma \in \Sigma_{k}} V_{\sigma(1)} \otimes_{R} \ldots \otimes_{R} V_{\sigma(k)}
$$

for $k, n, p \in \mathbb{N}$. He claims that these operations satisfy the axioms of power operations given in section 7 of [Gr1] (for checking this see also [GV]) and similarly to the above construction he obtains simplicial maps

$$
\Lambda^{k}: G \mathcal{M}^{\Sigma_{n}} \rightarrow \mathcal{H}^{k} \mathcal{M}^{\Sigma_{n k}}, \quad k, n \in \mathbb{N}
$$

It is easy to see that for $n=1$ and $k=l$ this map $\Lambda^{k}$ agrees with the above $l$-th shuffle power $\otimes^{l}: G \mathcal{M} \rightarrow \mathcal{H}^{l} \mathcal{M}^{\Sigma_{l}}$.

Now let $a \in \mathbb{Z} / l \mathbb{Z}$ and let $F_{a}: \mathcal{M}^{\Sigma_{l}} \stackrel{\text { can }}{\longrightarrow} \mathcal{M}^{C \rtimes G} \longrightarrow \mathcal{M}$ be the exact functor constructed in section 1 . We call the simplicial map

$$
\begin{aligned}
{[a]_{l}: G \mathcal{M} } & \rightarrow \mathcal{H}^{l} \mathcal{M} \\
\operatorname{Exact}(\Gamma(A), \mathcal{M}) \ni M & \mapsto F_{a} \circ M^{\otimes l} \in \operatorname{Exact}\left(\Gamma^{l}(A), \mathcal{M}\right)
\end{aligned}
$$

( $A \in$ Ord) the cyclic $l$-th shuffle power with eigenvalue $\zeta_{l}^{a}$.

In section 7 of [Gr1] (using a certain subdivision procedure) Grayson defines a certain continuous map

$$
\Xi_{l}:\left|\mathcal{H}^{l} \mathcal{M}\right| \rightarrow\left|G^{l} \mathcal{M}\right|
$$

from the geometric realization of $\mathcal{H}^{l} \mathcal{M}$ to the geometric realization of a certain $l$-simplicial set $G^{l} \mathcal{M}$ which in turn is naturally homotopy equivalent to $G \mathcal{M}$.

Definition. The composition

$$
|G \mathcal{M}| \stackrel{\left|[a]_{l}\right|}{\longrightarrow}\left|\mathcal{H}^{l} \mathcal{M}\right| \stackrel{\Xi_{l}}{\longrightarrow}\left|G^{l} \mathcal{M}\right|
$$

of the geometric realization $\left|[a]_{l}\right|$ of $[a]_{l}$ with the continuous map $\Xi_{l}$ is called the cyclic $l$-th power with eigenvalue $\zeta_{l}^{a}$ and will be denoted by $[a]_{l}$ again. For any $q \geq 0$ we give the same name to the map

$$
[a]_{l}: K_{q}(R \# \Gamma) \rightarrow K_{q}(R \# \Gamma)
$$

induced by $[a]_{l}$ on the $q$-th homotopy groups. The difference $\psi^{l}:=[0]_{l}-[1]_{l}$ is called the $l$-th Adams operation on $K_{q}(R \# \Gamma)$.

Proposition 8. The $l$-th Adams operation on $K_{0}(R \# \Gamma)$ defined here agrees with the $l$-th Adams operation defined in section 2.

Proof. This immediately follows from the "minus part" of the following lemma.

Lemma 5. For all finitely generated projective $R \# \Gamma$-modules $V, W$ we have

$$
[a]_{l}(V \pm W)=V[a]_{l} \pm W[a]_{l}+\frac{(V \pm W)^{l}-V^{l} \mp W^{l}}{l} \quad \text { in } \quad K_{0}(R \# \Gamma) .
$$

In particular for $l \neq 2$ we have $[a]_{l}(-W)=-W[a]_{l}$ in $K_{0}(R \# \Gamma)$.

Proof. First we consider the "minus part" of this formula. Let

$$
M: \Gamma(\{0\}) \rightarrow \mathcal{M}, \quad \frac{0}{L} \mapsto V, \quad \frac{0}{R} \mapsto W
$$


be the 0 -simplex of $G \mathcal{M}$ corresponding to $V-W$. Similarly to section 8 of [Gr1] one easily shows:

$$
[a]_{l}(V-W)=\sum_{\substack{a+b_{1}+\ldots+b_{u}=l \\ a \geq 0, b_{1}, \ldots, b_{u} \geq 1}}^{(-1)^{u} F_{a}\left(M^{\otimes l}\left(\alpha\left(a, b_{1}, \ldots, b_{u}\right)\right)\right)}
$$

where

$$
\alpha\left(a, b_{1}, \ldots, b_{u}\right):=(\underbrace{\frac{0}{L}, \wedge, \ldots, \wedge, \frac{0}{L}}_{a}, \otimes, \underbrace{\frac{0}{R}, \wedge, \ldots, \wedge, \frac{0}{R}}_{b_{1}}, \otimes, \ldots, \otimes, \underbrace{\frac{0}{R}, \wedge, \ldots, \wedge, \frac{0}{R}}_{b_{u}}) \in \Gamma^{l}(A) .
$$

By the construction of the $l$-th shuffle power we have

$$
M^{\otimes l}\left(\alpha\left(a, b_{1}, \ldots, b_{u}\right)\right)=\underset{\tilde{\sigma} \in \Sigma_{l} / \sim_{\alpha}}{\oplus} M\left(\frac{0}{\tilde{\sigma}(1)}\right) \otimes \ldots \otimes M\left(\frac{0}{\tilde{\sigma}(l)}\right)
$$

where $\tilde{\sigma}(i)=L$, if $\sigma(i) \leq a$ for one (and then for all) $\sigma \in \tilde{\sigma}$, and $\tilde{\sigma}(i)=R$ else. Like in the proof of Proposition 3 for all $\left(a, b_{1}, \ldots, b_{u}\right) \neq(l)$ and $\neq(0, l)$ the cycle $\langle 1, \ldots, l\rangle$ acts without fixed points on $\Sigma_{l} / \sim_{\alpha}$ and the module $F_{a}\left(M^{\otimes l}\left(\alpha\left(a, b_{1}, \ldots, b_{u}\right)\right)\right)$ is independent of $a \in \mathbb{Z} / l \mathbb{Z}$.

On the other hand by the Corollary of Theorem 4.2 in [Ko2] the power $(V-W)^{l}$ may be computed via the $(1, \ldots, 1)$-shuffle product, i. e. similarly as above we obtain

$$
\begin{gathered}
(V-W)^{l}=V^{l}-W^{l}+\sum_{\left(a, b_{1}, \ldots, b_{u}\right) \neq(l) \text { and } \neq(0, l)}(-1)^{u} M^{\otimes l}\left(\alpha\left(a, b_{1}, \ldots, b_{u}\right)\right) \\
=V^{l}-W^{l}+\sum_{\left(a, b_{1}, \ldots, b_{u}\right) \neq(l) \text { and } \neq(0, l)} l(-1)^{u} F_{a}\left(M^{\otimes l}\left(\alpha\left(a, b_{1}, \ldots, b_{u}\right)\right)\right) .
\end{gathered}
$$

Here the second equality follows from Proposition 1 and the fact that $F_{a}\left(M^{\otimes l}\left(\alpha\left(a, b_{1}, \ldots, b_{u}\right)\right)\right)$ is independent of $a \in \mathbb{Z} / l \mathbb{Z}$. Together with the above formula for $[a]_{l}(V-W)$ this shows the "minus part" of the lemma. The "plus part" can be deduced in a similar fashion from the formula

$$
[a]_{l}(V+W)=\sum_{\lambda=0}^{l} F_{a}\left(M^{\otimes l}(\underbrace{\frac{0}{L}, \wedge, \ldots, \wedge, \frac{0}{L}}_{\lambda}, \otimes, \underbrace{\frac{1}{0}, \wedge, \ldots, \wedge, \frac{1}{0}}_{l-\lambda})\right)
$$

(cf. the remark in parentheses in the proof of Proposition 3). Here $M$ denotes the 1-simplex of $G \mathcal{M}$ given by $M\left(\frac{0}{L}\right)=M\left(\frac{0}{R}\right)=V, M\left(\frac{1}{L}\right)=M\left(\frac{1}{R}\right)=V \oplus W$, and $M\left(\frac{1}{0}\right)=W$.

Corollary. For all $x, y \in K_{0}(R \# \Gamma)$ we have

$$
[a]_{l}(x+y)=[a]_{l}(x)+[a]_{l}(y)+\frac{(x+y)^{l}-x^{l}-y^{l}}{l} \text { in } \quad K_{0}(R \# \Gamma) .
$$

Proof. We may write $x=V-W, y=V^{\prime}-W^{\prime}$ with finitely generated, projective $R \# \Gamma$ modules $V, W, V^{\prime}, W^{\prime}$. Then using Lemma 5 we obtain

$$
[a]_{l}(x+y)=[a]_{l}\left(V-W+V^{\prime}-W^{\prime}\right)=[a]_{l}\left(V \oplus V^{\prime}-W \oplus W^{\prime}\right)
$$




$$
\begin{aligned}
& =[a]_{l}\left(V \oplus V^{\prime}\right)-[a]_{l}\left(W \oplus W^{\prime}\right)+\frac{\left(V \oplus V^{\prime}-W \oplus W^{\prime}\right)^{l}-\left(V \oplus V^{\prime}\right)^{l}+\left(W \oplus W^{\prime}\right)^{l}}{l} \\
& =[a]_{l}(V)+[a]_{l}\left(V^{\prime}\right)-[a]_{l}(W)-[a]_{l}\left(W^{\prime}\right)+\frac{\left(V+V^{\prime}-W-W^{\prime}\right)^{l}-V^{l}-V^{\prime l}+W^{l}+W^{\prime l}}{l} \\
& =[a]_{l}(V-W)+[a]_{l}\left(V^{\prime}-W^{\prime}\right)+\frac{\left(V+V^{\prime}-W-W^{\prime}\right)^{l}-(V-W)^{l}-\left(V^{\prime}-W^{\prime}\right)^{l}}{l} \\
& =[a]_{l}(x)+[a]_{l}(y)+\frac{(x+y)^{l}-x^{l}-y^{l}}{l} .
\end{aligned}
$$

Remark. In order to show that exterior powers induce well-defined maps $\lambda_{k}: K_{0}(R) \rightarrow$ $K_{0}(R), k \geq 1$, one usually shows that the association $V \mapsto \sum_{k \geq 0}\left[\Lambda^{k} V\right] t^{k}$ extends to a welldefined homomorphism $K_{0}(R) \rightarrow 1+t K_{0}(R)[[t]]$. I don't know such a simple argument for the operations $[a]_{l}, a \in \mathbb{Z} / l \mathbb{Z}$, on $K_{0}(R \# \Gamma)$. However, one can show directly (i. e. without using Grayson's techniques) that the map $[a]_{l}$ defined by

$$
[a]_{l}\left(\sum_{i} n_{i} V_{i}\right):=\sum_{i} n_{i} V_{i}[a]_{l}+\frac{\left(\sum n_{i} V_{i}\right)^{l}-\sum n_{i} V_{i}^{l}}{l}
$$

on the free abelian group with basis the isomorphism classes of finitely generated, projective $R \# \Gamma$-modules factorizes through the Grothendieck group $K_{0}(R \# \Gamma)$.

The following considerations are concerned with the question how to prove that the Adams operation $\psi^{l}$ commutes with the Cartan homomorphism $c: K_{q}(R \# \Gamma) \rightarrow K_{q}(\Gamma, R)$. Here $K_{q}(\Gamma, R)$ denotes Quillen's $q$-th $K$-group associated with the exact category of finitely generated, $R$ projective $R \# \Gamma$-modules and $\psi^{l}$ on $K_{q}(\Gamma, R)$ is defined via the $l$-th Newton polynomial in the exterior power operations $\lambda_{1}, \ldots, \lambda_{l}$ on $K_{q}(\Gamma, R)$ which in turn were constructed by Grayson in [Gr1]. As above for any $a \in \mathbb{Z} / l \mathbb{Z}$ and any $q \geq 0$ we can define the cyclic $l$-th power $[a]_{l}$ on $K_{q}(\Gamma, R)$.

Lemma 6. The operations $[0]_{l}$ and $[1]_{l}$ on $K_{0}(\Gamma, R)$ are natural transformations of $\lambda$-rings, i. e. they can be expressed as polynomials in the exterior power operations $\lambda_{k}, k \geq 1$.

Proof. We view the symmetric polynomials

$C_{l, 1}:=\frac{\left(t_{1}+\ldots+t_{l}\right)^{l}-\left(t_{1}^{l}+\ldots+t_{l}^{l}\right)}{l} \quad$ and $\quad C_{l, 0}:=\left(t_{1}+\ldots+t_{l}\right)^{l}-(l-1) C_{l, 1} \in \mathbb{Z}\left[t_{1}, \ldots, t_{l}\right]$

as polynomials in the elementary symmetric polynomials $\lambda_{1}:=t_{1}+\ldots+t_{l}, \ldots, \lambda_{l}:=t_{1} \cdot \ldots \cdot t_{l}$ and claim that

$$
[1]_{l}=C_{l, 1}\left(\lambda_{1}, \ldots, \lambda_{l}\right) \quad \text { and } \quad[0]_{l}=C_{l, 0}\left(\lambda_{1}, \ldots, \lambda_{l}\right) \quad \text { on } \quad K_{0}(\Gamma, R)
$$

where now $\lambda_{1}, \ldots, \lambda_{l}$ are the exterior power operations. Obviously we have

$$
C_{l, 1}=\frac{\lambda_{1}^{l}-\psi^{l}}{l} \quad \text { and } \quad C_{l, 0}=\frac{\lambda_{1}^{l}+(l-1) \psi^{l}}{l}
$$

and Lemma 5 holds for $K_{0}(\Gamma, R)$ with $[a]_{l}$ replaced by $C_{l, 0}$ or $C_{l, 1}$. Hence like in the proof of Proposition 4 by the geometric splitting principle it suffices to show $[0]_{l}(V)=C_{l, 0}(V)$ and $[1]_{l}(V)=C_{l, 1}(V)$ in $K_{0}(\Gamma, R)$ for any $R \# \Gamma$-module $V$ which is projective of rank 1 over $R$. But this follows from (the proof of) Example 3. 
Now, in order to show that $\psi^{l}$ commutes with the Cartan homomorphism $c: K_{q}(R \# \Gamma) \rightarrow$ $K_{q}(\Gamma, R)$ it suffices to show that

$$
[1]_{l}=C_{l, 1}\left(\lambda_{1}, \ldots, \lambda_{l}\right) \quad \text { and } \quad[0]_{l}=C_{l, 0}\left(\lambda_{1}, \ldots, \lambda_{l}\right) \quad \text { on } \quad K_{q}(\Gamma, R) .
$$

This is certainly true for $l=2$ because $[1]_{2}=\lambda_{2}$ and $[0]_{2}$ is the symmetric square $\sigma_{2}$ which in turn equals $\lambda_{1}^{2}-\lambda_{2}$ by Theorem 6.1 of [Ko2].

For $l=3$ one can show that for any finitely generated, $R$-projective $R \# \Gamma$-module $V$ the sequence

$$
0 \rightarrow \Lambda^{3} V \rightarrow \Lambda^{2} V \otimes V \rightarrow V[1]_{3} \rightarrow 0
$$

is exact. Here the first arrow comes from the Koszul complex and the second arrow is the composition of the natural embedding $\Lambda^{2} V \otimes V \hookrightarrow V^{\otimes 3}$ with the projection $V^{\otimes 3} \rightarrow V[1]_{3}$ coming from Proposition 1. Furthermore this sequence can be completed to a diagram as in Fig. 2 of [Ko2]. Like there one can deduce from this that $[1]_{3}=\lambda_{1} \cdot \lambda_{2}-\lambda_{3}$ already on the simplicial level. Together with the fact

$$
(l-1)[1]_{l}+[0]_{l}=\lambda_{1}^{l}
$$

coming from Proposition 1 and Example 1b) this shows the above claims for $l=3$.

For $l>3$ the essential problem is to find (universal) exact sequences which relate the cyclic power $[1]_{l}$ to exterior powers. The previous examples make it not unreasonable to conjecture that for any finitely generated, $R$-projective $R \# \Gamma$-module $V$ there is a (universal) resolution

$$
0 \rightarrow D_{1} V \rightarrow D_{2} V \rightarrow \ldots \rightarrow D_{l-1} V \rightarrow V[1]_{l} \rightarrow 0
$$

of $V[1]_{l}$ where for each $r=1, \ldots, l-1 D_{r} V$ is the following direct sum of tensor products of exterior powers of $V$ :

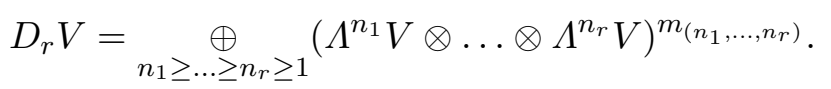

Here $(-1)^{l-r+1} m_{\left(n_{1}, \ldots, n_{r}\right)}$ is the coefficient of the monomial $\lambda_{1}^{n_{1}} \cdot \ldots \cdot \lambda_{r}^{n_{r}}$ in the polynomial $C_{l, 1}$. This coefficient can be rather easily computed by the formula

$$
C_{l, 1}=\frac{\lambda_{1}^{l}-\psi^{l}}{l}=\frac{\lambda_{1}^{l}-\sum_{i=1}^{l}(-1)^{i-1} i \lambda_{i} \cdot s_{l-i}}{l}
$$

(cf. section 3 of [Gr2]). In particular, one can deduce from this formula that the multiplicity $m_{\left(n_{1}, \ldots, n_{r}\right)}$ is positive for all $r=1, \ldots, l-1$ and all $n_{1} \geq \ldots \geq n_{r}$ with $n_{1}+\ldots+n_{r}=l$, and zero otherwise (e. g. $D_{1} V=\Lambda^{l} V, D_{l-1} V=\Lambda^{2} V \otimes V \otimes \ldots \otimes V$ ). There is a canonical choice for the map $D_{l-1} \rightarrow V[1]_{l}$ in the above resolution, namely the composition of the canonical embedding $\Lambda^{2} V \otimes V \otimes \ldots \otimes V \hookrightarrow V^{\otimes l}$ with the projection $V^{\otimes l} \rightarrow V[1]_{l}$ coming from Proposition 1. One can show that this composition is surjective. Thus the remaining problem is to construct (universal) homomorphisms, $D_{r} \rightarrow D_{r+1}, r=1, \ldots, l-2$, which make the above sequence exact. For instance, for $l=5$ one should be able to define arrows in the sequence

$$
\begin{array}{r}
0 \rightarrow \Lambda^{5} V \rightarrow \Lambda^{4} V \otimes V \oplus \Lambda^{3} V \otimes \Lambda^{2} V \rightarrow \Lambda^{3} V \otimes V \otimes V \oplus \Lambda^{2} V \otimes \Lambda^{2} V \otimes V \\
\rightarrow \Lambda^{2} V \otimes V \otimes V \otimes V \rightarrow V[1]_{5} \rightarrow 0
\end{array}
$$

such that this becomes an exact sequence and one should be able to generalize this sequence like in Fig. 1 and Fig. 2 of section 6 of [Ko2]. 
Remark. Obviously, using standard techniques one can prove that the Adams operation $\psi^{l}$ and the cyclic $l$-th powers $[a]_{l}, a \in \mathbb{Z} / l \mathbb{Z}$, commute with the base change homomorphism $K_{q}(R \# \Gamma) \rightarrow K_{q}\left(R^{\prime} \# \Gamma\right)$ with respect to any homomorphism $R \rightarrow R^{\prime}$ of $\Gamma$-rings. If the characteristic of $R$ is a prime number $p$ one can define $\psi^{p}$ on $K_{q}(R \# \Gamma)$ to be the base change homomorphism $F^{*}$ with respect to the Frobenius endomorphism $F$. Then $\psi^{p}$ commutes with each $\psi^{l}$. However, in general the usual problems enter the picture when one wants to show that the Adams operations constructed above commute with each other and that they are multiplicative (on the simplicial level). Nevertheless, using a fixed ordering of the prime numbers, one can define an Adams operation $\psi^{k}$ on $K_{q}(R \# \Gamma)$ for each $k \in \mathbb{N}$ which is invertible in $R$.

\section{References}

[At] M. F. Atiyah, Power operations in K-theory, Quart. J. Math. 17 (1966) 165-193.

[Be] D. J. Benson, Lambda and psi operations on Green rings, J. Algebra 87 (1984) 360-367.

[BC] D. Burns and T. Chinburg, Adams operations and integral Hermitian-Galois representations, Preprint, 1994.

[CNT] Ph. Cassou-Noguès and M. J. Taylor, Opérations d'Adams et Groupe des classes d'Algèbre de groupe, J. Algebra 95 (1985) 125-152.

[Dr] A. Dress, One more shortcut to Galois theory, Adv. in Math. 110 (1995) 129-140.

[F] A. Fröhlich, Galois module structure of algebraic integers, Ergebnisse der Mathematik und ihrer Grenzgebiete, 3. Folge, Band 1 (Springer, New York, 1983).

[FL] W. Fulton and S. Lang, Riemann-Roch algebra, Grundlehren der mathematischen Wissenschaften, Vol. 277 (Springer, New York, 1985).

[GG] H. Gillet and D. Grayson, The loop space of the $Q$-construction, Illinois J. Math. 31 (1987) 574-597.

[Gr1] D. Grayson, Exterior power operations on higher $K$-theory, $K$-Theory 3 (1989) 247-260.

[Gr2] D. Grayson, Adams operations on higher $K$-theory, $K$-Theory 6 (1992) 97-111.

[Gre] C. Greither, Cyclic Galois extensions of commutative rings, Lecture Notes in Mathematics, Vol. 1534 (Springer, New York, 1992).

[GV] T. Gunnarsson and R. Schwänzl, Operations in $A$-theory, Preprint, 1994.

[Ke] M. Kervaire, Opérations d'Adams en Théorie des représentations linéaires des groupes finis, Enseign. Math. 22 (1976) 1-28.

[Ko1] B. Köck, Das Adams-Riemann-Roch-Theorem in der höheren äquivarianten $K$-Theorie, J. Reine Angew. Math. 421 (1991) 189-217.

[Ko2] B. Köck, Shuffle products in higher K-theory, J. Pure Appl. Algebra 92 (1994) 269-307.

[Ko3] B. Köck, The Grothendieck-Riemann-Roch theorem in the higher $K$-theory of group scheme actions, in preparation. 